\title{
Overcoming barriers to hepatitis C elimination
}

\section{doi:10.1136/flgastro-2018-101114}

Recent advances in the treatment of hepatitis $\mathrm{C}$ virus (HCV) infection have produced highly effective all-oral directacting antivirals (DAA) therapies with minimal side effects and short treatment duration. This is encouraging news for the 71 million persons estimated to be chronically infected with HCV worldwide. ${ }^{1}$ From a public health perspective, HCV prevalence shall be eliminated if available treatment is also targeted to those who most likely transmit the virus. Despite this scientific breakthrough, a systematic review from the USA described that of $43 \%$ of patients are aware of their HCV diagnosis, only $16 \%$ had begun treatment. ${ }^{2}$ Evidently, long-standing barriers to treatment need to be addressed for these effective therapies to be delivered. Therefore, in the DAA era, where treatment efficacy, side effects and duration are not a problem, identification of barriers that prevent the delivery of HCV care is an important issue that remains pending. Historically, multiple-layered barriers related to a patient, provider and structural factors may effectively prevent HCV linkage to care (figure 1 ):

\section{Patient barriers}

Patient-related factors are a common source of treatment deferral and limited awareness, poor adherence to physician recommendations and treatment fears.

\section{Manuel Mendizabal, ${ }^{\oplus 1,2}$ Cristina Alonso, ${ }^{1,2}$ Marcelo O Silva ${ }^{1,2}$}

Patient unawareness of HCV infection represents one of the greatest obstacles to treatment. Efforts to scale up $\mathrm{HCV}$ diagnosis must include community education and stigma issues. Confusion about disease complications and modes of transmission is also common. Once $\mathrm{HCV}$ diagnosis is established, patients frequently fail to seek treatment probably because they may not recognise the urgency to treat this asymptomatic infection. In some regions, patients with HCV are more likely to be uninsured when compared with persons without HCV. Uninsured individuals are less likely to have regular visits with healthcare professionals, reducing the probability of diagnosis and treatment. Furthermore, patients might feel stigmatised because of the association between HCV and substance abuse or HIV. Fears of treatment were historically a well-described barrier in the interferon era given the treatment-related side effects. Providers should educate and counsel patients about interferon-free regimens to allay such fears.

\section{Structural barriers}

There is a marked regional variation concerning deficits in provider knowledge. In some countries, physicians may have limited HCV treatment experience and do not recognise important treatment principles. The development of DAAs has resulted in the need for significant expansion in the number of providers who can treat HCV. While primary care physicians (PCP) have always have a role in detection and counselling for HCV, their scope of practice is currently being expanded to include $\mathrm{HCV}$ treatment. Several successful HCV training programmes have described equivalent sustained virological response (SVR) rates whether patients were treated by specialist physicians or PCP. ${ }^{3}$

\section{Provider barriers}

Strategies have been implemented to increase identification of $\mathrm{HCV}$-infected individuals who remained undiagnosed. In 2012, the Centers for Disease Control promoted the recommendation that all individuals born between 1945 and 1965 should have one-time HCV screening test. In the Americas, there are $>7.2$ million individual with $\mathrm{HCV}$ viraemia, and 1.2 million persons living with HCV-related cirrhosis. ${ }^{3}$ At least a half of whom belong to Latin America and the Caribbean countries. This is a region mostly composed by low-income and middle-income countries, where access to diagnosis and linkage to care are worse than the reported in the USA and Canada. ${ }^{3}$ To improve access to care, Pharma companies and World Trade Organisation (WTO) allowed low-income countries to have access to new treatments through appropriate transfer technology (generic) policies. This 


\begin{tabular}{|c|c|c|}
\hline Layer & Barriers & Proposed solution \\
\hline Patient & $\begin{array}{l}\text { - Access to healthcare professionals } \\
\text { - } \text { Competing health priorities } \\
\text { - } \text { Poor HCV knowledge } \\
\text { - } \text { Asymptomatic infection } \\
\text { - } \text { Fears about treatment } \\
\text { HCV stigma }\end{array}$ & $\begin{array}{l}\text { - } \text { Community education } \\
\text { - } \text { Engage communities to promote } \\
\text { - Creating } \\
\text { - }\end{array}$ \\
\hline Providers & $\begin{array}{ll}\text { - } & \text { Limited knowledge } \\
\text { - } & \text { Treatment misconceptions }\end{array}$ & $\begin{array}{l}\text { - } \text { Training programs } \\
\text { - } \text { Simplify model of care } \\
\text { Decentralise HCV care to primary } \\
\text { care }\end{array}$ \\
\hline Structural & $\begin{array}{l}\text { - Insufficient providers who can treat HCV } \\
\text { - National treatment guidelines } \\
\text { - Unaffordable diagnostic tests } \\
\text { - } \text { HCV medication high price } \\
\text { - Lack of national/regional HCV strategies }\end{array}$ & $\begin{array}{l}\text { - Strong political commitment } \\
\text { - } \text { Funding to start HCV programs } \\
\text { - } \text { Decrease cost of care } \\
\text { - Estimate disease burden } \\
\text { - Identify high risk groups }\end{array}$ \\
\hline
\end{tabular}

Figure 1 Overcoming barriers to increase access to hepatitis $C$ treatment. $\mathrm{HCV}$, hepatitis $\mathrm{C}$ virus.

advantage is not available for middle-income countries that remain in a limbo or purgatory from an access perspective. This inequity needs to be considered when discussing WHO goals for HCV elimination for 2030. WHO goals will be unachievable if this barrier to middle-income countries is not modified. Moreover, Latin America's hospital-centred segmented and fragmented health systems are another structural barrier. This structural barrier limits outreach and delivery of an integrated and systematic response to the disease burden. According to this PAHO review, $<50 \%$ of countries in the Americas have active Viral Hepatitis Programmes under the Ministry of Health.

\section{How can we overcome treatment barriers?}

There is a need to improve diagnosis, access to care and treatment of HCV infection, especially in resource-limited settings. Key priorities for scaling up HCV treatment include developing models for integrating $\mathrm{HCV}$ management into primary care, reducing the cost of care and increasing political commitment. Many of these obstacles have been addressed for HIV treatment and a number of lessons can be drawn to help improve access to HCV care. ${ }^{4}$

Historically, specialist physicians (eg, hepatologists and infectious disease physicians) have managed patients with HCV. Lack of access to specialty care services at community-based health centres is still a major problem. Fortunately, in recent years, we have experienced the approval of novel technologies that can simplify HCV model of care: (i) provision of easy-to-use assays for serological and virological analyses (pointof-care assays) that facilitate screening, exclude co-infections and allow treatment monitoring. ${ }^{5}$ Dried blood spots can be a feasible and reliable test for viral hepatitis. (ii) Non-invasive assessment of liver fibrosis has also presented major advances in the last decade. These range from serological markers such as aspartate aminotransferase to platelet ratio index (APRI) and portable transient elastography (TE) (eg, Fibroscan). ${ }^{6}$ The implementation of these non-invasive methods can avoid the use of a liver biopsy for mass screening, a stressful and invasive procedure. Both APRI and TE perform well in the identification of cirrhosis or no fibrosis, but they perform less well in resolving intermediate degrees of fibrosis. Patients with fibrosis grades 3 or 4 should promptly receive antiviral therapy in order to prevent the development of liver-related complications. ${ }^{7}$ Thus, when patients present an APRI score $>2.0$ or $\mathrm{TE}>10 \mathrm{kPa}$ DAA therapy is strongly recommended. In settings where treatment expands outside of specialty clinics or in low-income and middle-income countries, APRI is generally available, cheap and simple, and the information it provides is reliable. However, staging of liver fibrosis is not relevant for addressing treatment indication. WHO, European Association for the Study of the Liver (EASL), American Associacion for the Study of Liver Diseases (AASLD), Asian-Pacific Association for the Study of the Liver (APASL) and Latin American Association for the
Study of the Liver (ALEH) guidelines recommend that all patients should be treated regardless their stage of fibrosis. Nevertheless, liver fibrosis staging remains critical to define patient's length of therapy and follow-up. (iii) The development of new DAAs has greatly helped to simplify and standardise patient care. New HCV treatment guidelines which can be easily accessed online or by mobile phone applications are a useful reference tool.

All these advances have facilitated HCV treatment in some regions, making HCV elimination a realistic goal. In this issue of Frontline Gastroenterology, the group of Prof. O'Beirne from the University of the Sunshine Coast in Australia developed an interesting and successful HCV model of care addressing the challenge of providing treatment to patients in remote areas. ${ }^{8}$ Australia is a vast country with a strong political commitment in achieving WHO goal of HCV elimination. Thus, DAA are freely available to all Australians with $\mathrm{HCV}$ infection regardless their disease stage, and all medical practitioners are able to prescribe DAA. In order to facilitate linkage to care the University of Sunshine Coast developed two models of HCV treatment. The first model allowed a rapid access to HCV clinic for patients referred by their PCP. A hepatologist and nurse evaluated patients and a recommendation was made with a checklist for follow-up. Patients underwent treatment in primary care facilities and nurses contacted the patients to ensure treatment indications and determine SVR date. The second model consisted 
in two nurses equipped with mobile Fibroscan providing TE-based assessment in regional healthcare centres, including a large prison. Following assessment, patients were discussed at a weekly multidisciplinary team where recommendations were made. Interestingly, authors compared APRI with contemporaneous TE. A total of 327 patients were treated in primary care and no significant differences in SVR rates were seen when compared with those treated in secondary care. Furthermore, they validated APRI (cutoff $<1$ ) to identify patients with very low risk of cirrhosis. This hepatology partnership models appears to be highly effective at providing treatment advice to PCP and in evaluating liver fibrosis stage.

Australia has laid the foundation for HCV elimination with a strong political commitment based on high levels of screening and diagnosis, unrestricted access to DAA therapy and a diverse range of models of care. However, expanding access for HCV treatment in resource-limited settings requires a continuous effort to overcome practical and political challenges, a different WHO and WTO perspective should be contemplated for middle-income countries. The biggest challenge remains for developing countries with scarce specialist physicians and prohibited HCV treatment costs.

Contributors MM: wrote the manuscript, CA and MOS: revised and rewrote some sections of the text. MOS: provided content guideline and references.

Competing interests None declared. Patient consent for publication Not required.

Provenance and peer review Not commissioned; externally peer reviewed.

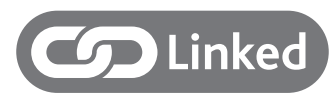

http://dx.doi.org/10.1136/flgastro-2018-101049

\section{REFERENCES}

1 World Health Organization. Global Hepatitis report. 2017 www.who.int. http://www.who.int/publications/globalhepatitis-report2017/en/ (Accessed 11 Dec 2018).

2 Yehia BR, Schranz AJ, Umscheid CA, et al. The treatment cascade for chronic hepatitis $C$ virus infection in the United States: a systematic review and metaanalysis. PLoS One 2014;9:e101554.
3 Arora S, Thornton K, Bradford A. Access to care: Management of hepatitis $C$ viral infection in remote locations. Clin Liver Dis 2012;1:87-90.

4 Ford N, Singh K, Cooke GS, et al. Expanding access to treatment for hepatitis $\mathrm{C}$ in resource-limited settings: lessons from HIV/AIDS. Clin Infect Dis 2012;54:1465-72.

5 Johannessen A. Where we are with point-of-care testing. J Viral Hepat 2015;22:362-5.

6 European Association for Study of Liver, Asociacion Latinoamericana para el Estudio del Higado. EASL-ALEH Clinical Practice Guidelines: Noninvasive tests for evaluation of liver disease severity and prognosis. Journal of Hepatology 2015;63:237-64.

7 European Association for the Study of the Liver. Electronic address: easloffice@easloffice.euEuropean Association for the Study of the Liver. EASL Recommendations on Treatment of Hepatitis C 2018. J Hepatol 2018;69:461-511.

8 White L, Azzam A, Burrage L, et al. Facilitating treatment of $\mathrm{HCV}$ in primary care in regional Australia: closing the access gap. Frontline Gastroenterol 2019;10:210-6. 